\title{
Research of Toy Design Techniques Based on Virtual Reality
}

\author{
Li Fang \\ Suzhou Art \& Design Technology Institute, China \\ e-mail:lifang1213@163.com
}

\begin{abstract}
This paper first introduces the concept and allocation status of virtual reality technology, by analyzing the current computer-aided design problems in the toy, this paper also describes the virtual reality techniques advantages in toy design, mainly referring to improve the innovation of design, shorten the design process and improve design efficiency, reduce design costs and enhance the value of toys. Based on virtual reality technology, this paper gives the main toy design process, including construction of three-dimensional model, construction and interaction of virtual reality scenes and engineering drawing, mainly giving the introduction of VRML and Pro/E. Finally, the problems of virtual reality technology in the Chinese toy market are revealed. Once the problem is resolved, the virtual reality in the toy design will have a broader development prospects.
\end{abstract}

Keywords-Toy design; Virtual reality; VRML; Pro/E

\section{INTRODUCTION}

Virtual reality technology is to virtual out something and the environment that is not existing through a variety of digital technology, to make people feel like real scenes, allowing users to produce a feeling of immersion in the virtual environment visually. The virtual reality technology used in toys product design and development as well as the late show is called virtual toy design and presentation. Virtual toys design is to present the whole process of virtual implementation from conceptual design to be put into use on the computer constructed virtual environment, which is not only to make simulation and visualization simulation on the physical form of the product and manufacturing process, but also make prediction, evaluation, co-ordination and optimization on the toy products performance, behavior and functional value. The fundamental characteristics of virtual reality include immersion, interaction and imagination.

Virtual reality technology status in toy design and display applications mainly includes the following three aspects.

- Virtual prototyping replace physical prototypes

- Immersive display design

- Virtual Assembly

Researchers have increasingly turned to Virtual Reality Modeling Language (VRML) to represent toy design techniques. In VRML's early days, the result was a few toy examples that did not scale well, such as coarse, single-resolution elevation grids. Today, VRML is drawing more serious interest from researchers across the massive toy design field as the sidebar "Related Work" describes. Visualization tools have begun to expand into each other's domains such as geographic information system and others, and VRML offers cartographers and geographers the potential to disseminate 3D maps and spatial data over the World Wide Web. However, we have not seen useful large-scale VRML toy design databases to date. As an integrated discipline, Computer Aided Design (CAD) methodology mainly refers to tool design on the basis of computer and computer software. It is widely used in the fields of art which provides a new means and support for the design of art and opens a broader situation for the art development. Since its inception, colleges and universities at home and abroad have introduced it into the teaching of computer-aided design.

\section{THE STATUS AND EXISTING PROBLEMS OF TOY DESIGN WITH COMPUTER AID}

As to toy design, all that from the design results show to the drawing of production and processing are almost inseparable from the aid of computer, which is computer-aided design that we are talking about. Through the use of computer software, students create three dimensional renderings for artistic expression of three-dimensional modeling, and then enhance the effect of plane showing by the two-dimensional software, ultimately, use engineering drawing software to complete its process mapping. The use of computer-aided design not only shortens the design cycle, but also greatly reduces design costs, thereby improving the efficiency, which makes the design work closely follow the market trends. For example, for children toy design, after student determines design thinking sketches, three-dimensional renderings can be produced by computer-aided design software, and this process is not only necessary to prepare complicated traditional drawing tools, but also mimic a more realistic effect through the final rendering. Particularly important, the computer software making process is reversible, which can be modified and improved, thus greatly reduce the time cost of repeatedly drawn.

However, with its extremely rich and interactive features, the toy design is different from other product design. For the existing forms of reproduction, although computer-aided design breaks the limitations of two-dimensional renderings of the original performance. Through three-dimensional display, it allows users to understand the various features of the structure of the toy from different angle, but it is still inadequate for the use of state and interaction performance. When designers show 
their work, in addition to the three-dimensional renderings, it is usually accompanied by a large number of required text, which not only improves the difficulty of the user's understanding product information, but also leads users to understand bias and misuse because of the ambiguity of the text description.

\section{ADVANTAGES OF TOY DESIGN WITH VIRTUAL REALITY}

As a special product, as to toy's design elements, in addition to its shape, color, structure, functional ergonomics and environmental considerations, more need to focus on the interaction between users. A well-designed toys should not only "look good" but also be "fun", but also further achieve the purpose of entertaining, so the interaction and interface design becomes particularly important. Currently popular digital toys, such as digital pet toys and digital dolls, are arising new products expanding the interactive nature of the entity toys, and they can not only simulate the action and reaction of various real pet, but also achieve the interactive simulation in operation, also have the function of regulating emotion for the user, described as leading the fashion in toy design industry.

So in the manufacturing process of physical toy, how to take into account the effect of form shows, but also show the interaction between the toy and the player, not only represent a combination of static relationships between the structural components, but also achieve its entertainment dynamic relationship between the operation processes? Virtual reality technology with the main features of truth, immersive and interactive gives a solution to solve the above problem, to provide a new means and ways for toys design.

The concept of virtual reality is proposed by Jaron Lanier in US VPL in 1989, which generally refers to the use of computer software, hardware and related equipment to construct interactive virtual environment, it is not only able to provide users with immersion immersive environment but also allows the real-time feedback of the user visual, auditory, tactile and other sensory information to achieve real-time interaction between the user and the environment. The toys design based on virtual reality technology can take full advantage of the excellent interactive features of virtual reality technology, by simulating the interaction between toys and players in the process, providing an effective design reference for designers. In addition, by introducing the virtual reality technology into toy design teaching, it makes the teaching style of computer-aided design no longer a single performance, but demonstrate the use state of toy products. By doing so, on one hand, it can allow students to try to interact with the virtual model of the toy, personally experience the toy's using state and make user behavior assessment. On the other hand, through adequate classroom discussions and exchanges of the virtual toy model, make improvement and design evaluation, not only to achieve the purpose of teaching software skills but also improve students' ability to design thinking, which makes up the deficiencies of traditional computer-aided toy design teaching.

Virtual reality technology in toy design and development is significant, which can be shown in the following three aspects.

\section{A. Improve the Innovation of Design}

In recent years, CAD (Computer Aided Design) technology provides a strong support in the toy product development process, but we usually make a combination of multi software in virtual product design to achieve. For example, as to product modeling of special surface, we often use a combination of 3D Max, Rhino, and Pro/Engineer and other three-dimensional software to complete designing the virtual product model, which essentially did not free the designers from the mouse and keyboard, and the designers truly did not participate in the virtual design. With the use of virtual reality technology and multimedia dimensional imaging technology in toys' innovative development process, as well as the improvement in the operation of integrated computer technology and human-machine interface for scientific and technical personnel, it will enhance the enthusiasm and creativity of designers.

\section{B. Shorten the Design Process and Improve Design Efficiency}

After applying the virtual reality technology into toy design process, you can save past prototyping processes to shorten the product design cycle and improve design efficiency and reduce design costs. Many international large toy manufacturers have competed to introduce virtual reality technology in the design of toys, such as the US HASBRO Company and Japanese TAKARA TOMY Company. TAKARA TOMY Company introduce virtual reality into "Up" series toys in 2007, and launched a virtual showcase of the latest products on its official website, to provide the players with a new interactive platform, development and sales cycles are shortened largely.

\section{Reduce Design Costs and Enhance the Value of Toys}

Virtual reality technology can make virtual modeling, and also perform physical and dynamics simulation on the properties of toys to test toys reliability to reduce costs and improve efficiency and enhance the added value of the toy. For example, from 1988 to 1995, the Guangzhou Bai Yun Mountain company received official sales agency of deformed toys from the US Hasbro, the price of some of the classic Transformers toys is: Hornets 11 yuan, Dinobots 48 yuan, Megatron 108 yuan. According to a survey, according to the average consumption of 200 yuan per capita, that of 500 million consumer is 1 billion yuan, except tariffs, toy manufacturing costs and other expenses, conservatively said, its net profit should be around 500 million yuan. After the application of virtual reality technology in toy design and demonstration, in 2007, the US Hasbro globally release Transformers toy model of the film version, as of May 2008, its sales reached \$ 150 million.

\section{TOY DESIGN PROCESS BASED ON VIRTUAL REALITY}

The toy design based on virtual reality technology is on the basis of the traditional computer-aided design drafting, modeling and rendering performance. On the basis, simulate the virtual scene and use process, and enable users to interact with the toy with the mouse and keyboard to make true performance of the application form of toy 
products, so as to serve for toy design. On the basis of traditional toy design process, further extended to virtual reality technology, it can be divided into the following three steps: construction of three-dimensional model, construction and interaction of virtual reality scenes and engineering drawing. Next, this paper will describe the three design steps in detail.

\section{A. Construction of Three-dimensional Model}

Traditional toy design step usually based 3D modeling and renderings show as ending, and this stage is also a key step which is exactly convergence pass between traditional toy design and virtual reality toy design. The current mainstream of 3D modeling software mainly includes Maya and 3ds Max, whose constructed model can be exported into a virtual scene model. $3 \mathrm{ds}$ Max has a very powerful modeling capabilities and material performance capabilities, and its superior rendering capabilities can realistically build a toy design and modeling of the most difficult surface rendering hair plush toys, including the form, structure, size proportion, texture, color and other elements of design work to construct a three-dimensional model of the ideal.

For the traditional three-dimensional computer-aided design modeling is different, the model built for the virtual scene should try to streamline the number of its facets, that is, we usually say "Jane die", through adjustment of the normal vector to remove all invisible surface, take it to be able to perceive the visual effects as the standard elements which reduce the accuracy, try to improve the display speed of the model, so as to prepare to import the virtual scene for real-time interaction. After completion of the three-dimensional modeling, the first is to set the light in the virtual scene model, by adjusting the angle, intensity, reflectivity of illumination, in order to achieve optimal product mix and display results as the standard, set the scene lighting effects, and finally make three-dimensional model baking output.

\section{B. Construction and Interaction of Virtual Reality Scenes}

At present, for the interaction between user and the virtual scene making software mainly include Danish Unity3d, French Virtools and China's VRP and so on. The software integrates the functions of the various modules to achieve, designers can call the respective module, select the required components and easily build a virtual scene.

As an international standard virtual reality modeling language in use today, VRML is a universal programming platform. Through programming, designers can set the parameters of the virtual scene of model, to achieve real time interaction between the user and the virtual model according to certain qualification in the real world. These data can be used as a user experience used for designer to the next report on the improvement of the design as a reference.

VRML is based on WWW, interactive virtual reality modeling language. It provides a three-dimensional world and its internal basic description of the object. It is a high-level object-oriented language, which is similar to HTML language and a text-based language, and can run on multiple platforms. Basic principle of VRML is to use text message for three-dimensional scene description, Internet online transmission on the local machine by VRML browser interprets to generate three-dimensional scene, standards explaining the resulting is VRML specification, which is shown in Fig. 1.

The VRML file implementation process is shown as follow:

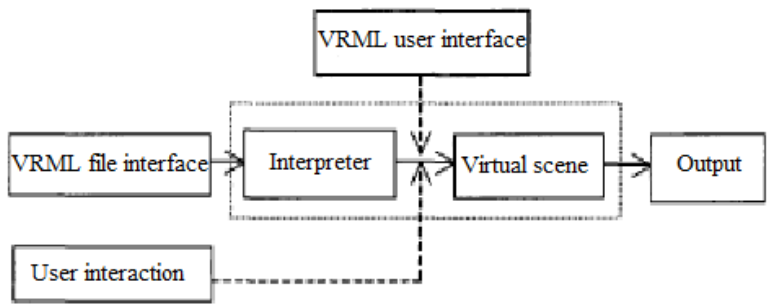

Figure. 1 VRML file implementation process

The latest VRML language compared to other 3D modeling languages has the following advantages: open, platform independence, low bandwidth requirements, based on the interaction of events, scalability, easily modeled.

\section{Engineering drawing}

After using virtual reality software to simulate and evaluate user scenarios, they can base on these report data for the pre-design works improvements. At the same time, using the initial three-dimensional model Maya and 3ds Max built, although the ratio may reflect the size of the toy between the various structural components, in terms of production for processing, these data is not enough. Therefore, after determining the final design of the toy program, they have to carry out the project for the processing and production system in order to achieve mass production and commercialization of the final product. Three-dimensional virtual model modeling has mainly two ways: one is the direct writing VRML code, another is to use three-dimensional modeling software for modeling, then creates another file into a VRML file format. As to Pro/Engineering modeling, using this method to create complex shapes is not only convenient but also visual.

Pro/E software is operated by the US Parametric Technology Corporation (PTC) with integration of three dimensional software CAD/CAM/CAE. Pro/Engineer software parameterization is known, which is the first application by Parametric Technology, and the current three-dimensional modeling software occupies an important position in the field. The new standard of Pro/Engineer is in the world mechanical CAD/CAE/CAM field and get the industry recognition and promotion, which is now one of the mainstream of $\mathrm{CAD} / \mathrm{CAM} / \mathrm{CAE}$ software, especially in the country to occupy an important position in the field of product design.

\section{PROBLEMS OF VIRTUAL REALITY TECHNOLOGY IN THE CHINESE TOY MARKET}

The development of virtual reality technology which is originated in the United States is late in China, there are many issues to be resolved in the development of virtual reality technology in the toy market in China. The first is the data exchange problem, now most manufacturing use CAD systems, and data of virtual design system is temporarily unable to directly transmit with CAD systems, which is the obstacle of many companies to use virtual reality technology. Followed by computer hardware problem, now there is a big gap between the hardware 
design of virtual reality and virtual reality technology requirements, such as the sensitivity of network, the transmission speed, data feedback system optimization and so on. Once again, it is financial problems, for a complete virtual reality system requires a lot of hardware and software support, many domestic manufacturers cannot invest huge amount of money, at present, these manufacturers still choose the traditional mode of operation orders, processing and export. These are the factors that limit and restrict the development of virtual reality technology in the Chinese toy market.

The current toys design of virtual reality is still in a preliminary stage and there are many unresolved issues. One is how to handle toys deformation due to external force and the other is how to solve the problem of emotional communication between users and toys. Once the problem is resolved, the virtual reality in the toy design will have a broader development prospects.

\section{CONCLUSIONS}

The application range of toy design based on virtual reality is very extensive. First, it breaks the static output restrictions of traditional computer-aided toy design and provides an interface interaction, allowing the designer to implement interaction from the user's point of experience and consideration in the design process, and simulate interactions between users and toys in order to continuously improve the design work. Second, the virtual reality toy provides a good medium for toy design professional classroom, teachers can point out the advantages and disadvantages of the design with a real-time demonstration, which not only makes comments more clear and specific, but also enhances the classroom activity, promoting good interaction between education and learning. Finally, it opens up a whole new field of professional teaching of toy design, by using VRML and other related web 3D technology, with the Internet to achieve multiplayer online teaching platform, which not only achieves distance learning but also achieves collaborative design environment.

\section{REFERENCES}

[1] M. Zyda, "From visual simulation to virtual reality to games." Computer, 38(9), pp.25-32, 2005.

[2] H.Y. Kan, D.G. Duffy and C.J. Su, "An Internet virtual reality collaborative environment for effective product design." Computers in Industry, 45(2), pp.197-213, 2001.

[3] K.S. Suh and Y.E. Lee, "The effects of virtual reality on consumer learning: an empirical investigation”. Mis Quarterly, 2005: 673-697.

[4] Liu C, Wang F, et al. Robust $\mathrm{H} \infty$ Control for Satellite Attitude Control System with Uncertainties and Additive Perturbation. International Journal of Science, 2014, 1(2): 1-9.

[5] Liu Y J, Ma C X, Zhang D L. EasyToy: plush toy design using editable sketching curves. IEEE Computer Graphics and Applications, 2009 (2): 49-57.

[6] Taborda E, Chandrasegaran S K, Kisselburgh L, et al. Enhancing visual thinking in a toy design course using freehand sketching//ASME 2012 International Design Engineering Technical Conferences and Computers and Information in Engineering Conference. American Society of Mechanical Engineers, 2012: 267-276.

[7] Reddy M, Leclerc Y, Iverson L, et al. TerraVision II: Visualizing massive terrain databases in VRML. Computer Graphics and Applications, IEEE, 1999, 19(2): 30-38.

[8] Belfore L A. An architecture support live updates and dynamic content in VRML based virtual worlds//In Military, Government, and Aerospace Symposium. 2002: 14-18.

[9] Liu C, Shi K, Wang F. Mass and mass center identification of target satellite after rendezvous and docking//Intelligent Control and Automation (WCICA), 2014 11th World Congress on. IEEE, 2014: 5802-5807.

[10] Reddy M, Leclerc Y G, Iverson L, et al. Modeling the digital earth in $\mathrm{vrml} / /$ PROC SPIE INT SOC OPT ENG. 2000, 3905: 113-121.

[11] Wang F. Mixed $\mathrm{H}_{2} / \mathrm{H}_{\infty}$ control for a satellite based on LMI. // Mechatronics and Control (ICMC), 2014 IEEE International Conference on. IEEE, 2014: 2114-2118.

[12] Rizzo A, Kim G. A SWOT analysis of the field of virtual reality rehabilitation and therapy. Presence, 2005, 14(2): 119-146.

[13] Chen J, Pu X, Yin X. The applications of virtual reality technology in terms of the design and display of toys. Science Technology Association Forum. 2008, 8: 101-102. 